EESTI NSV TEADUSTE AKADEEMIÁ TOIMETISED. 29. KÖIDË FUOSIKA * MATEMAATIKA. 1980, NR. 2

ИЗВЕСТИЯ АКАДЕМИИ НАУК ЭСТОНСКОН ССР. ТОМ 29 ФИЗИКА * МАТЕМАТИКА, 1980, Na 2

\title{
О СХОДИМОСТИ ТРАЕКТОРИЙ СКОЛЬЗЯЩЕГО ПЛАНИРОВАНИЯ В ДИНАМИЧЕСКОЙ МЕЖОТРАСЛЕВОИ МОДЕЛИ
}

\author{
(Представил Н. Алумяэ)
}

Рассмотрим динамическую модель

$$
A x_{t} \leqslant x_{t-1}, \quad x_{t} \geqslant 0, \quad t=1,2, \ldots,
$$

где $A$ - примитивная матрица технологических коэффициентов размерности $n \times n, x_{t}$ - вектор выпусков в момент $t$. Оптимальными назовем траектории, максимизирующие величину $u\left(x_{T}\right)$ на множестве допустимых траекторий $\left\{x_{t}\right\}_{0}^{T}$ при некотором фиксированном векторе начальных запасов $x_{0} \geqslant 0$, где $u$ - любая монотонно возрастающая (т. е. $u(x)>u(y)$, если $x>y)$ функция полезности, $T$ - плановый горизонт. Пусть $\alpha-$ максимальное по модулю собственное число матрицы $A, \hat{x}$ и $p$ - соответствующие ему нормированные правый и левый собственные векторы. По теореме Фробениуса-Перрона $\hat{x}>0, p>0$.

В [ $\left.{ }^{1}\right]$ для модели вида (1) определено понятие процесса скользящего (непрерывного) планирования: пусть $\left\{x_{t}(0)\right\}_{0}^{T}-$ произвольная оптимальная траектория при начальных запасах, равных $x_{0}$; из точки $x(1)=x_{1}(0)$, как начального состояния, вычисляем новую оптимальную траекторию $\left\{x_{t}(1)\right\}_{0}^{T}$, где $x_{0}(1)=x(1)$, и положим $x(2)=x_{1}(1)$. Продолжая процесс таким же образом, получим траекторию $\{x(\tau)\}_{0}^{\infty}$ с начальным состоянием $x(0)=x_{0}$, именуемую траекторией скользящего планирования или скользящим планом.

Из доказанного в $\left[{ }^{2}\right]$ следует, что для любого наперед заданного числа $\varepsilon>0$ всякая траектория скользящего планирования с достаточно длинным плановым горизонтом, исходящая из фиксированного начального состояния $x_{0}>0$, полностью лежит начиная с некоторого момента в конической $\varepsilon$-окрестности неймановского луча, т. е. в данном случае луча, порождаемого вектором $\hat{x}$. В то же время в $\left[{ }^{1}\right]$ приведен пример, показывающий, что, вообще говоря, траектория скользящего планирования может не сходиться к стационарному состоянию.

В настоящей работе сходимость (в смысле пропорций) скользящих планов к неймановскому лучу доказывается при некотором условии, налагаемом на принцип их составления, а именно: пусть оптимизация в (1) состоит в максимизации выпуска в конечный момент $T$ при фиксированных пропорциях продуктов, соответствующих исходному состоянию, т. е. на $k$-й итерации процесса скользящего планирования решается задача 


$$
\begin{gathered}
\max \left\{\gamma \mid A x_{t}(k) \leqslant x_{t-1}(k), x_{t}(k) \geqslant 0, t=1, \ldots, T\right. \\
\left.x_{T}(k) \geqslant \gamma x(k)\right\}, k=0,1, \ldots .
\end{gathered}
$$

Т е ор ема. Пусть $\{x(\tau)\}_{0}^{\infty}$ - произвольная траектория скользящего планирования в задаче (2), исходящая из некоторой точки $x_{0}>0$. Если плановый горизонт $T$ достаточно велик (не менее некоторого $T^{\prime}$ ), то найдется число с >0 такое, что

$$
\alpha^{\tau} x(\tau) \underset{\tau \rightarrow \infty}{\longrightarrow} c \hat{x} .
$$

Обозначим символом $\|\cdot\|$ норму в пространстве $R_{+}{ }^{n}, \varrho(x, y)=$ $=\|x /\| x\|-y /\| y\|\|$ - угловое расстояние между векторами $x$ и $y$, $q(x)=\min _{i=1, \ldots, n} x^{(i)} / \hat{x}^{(i)}$ для $x \geqslant 0$. Поскольку $p>0$, то для $x \geqslant 0$ можно, не умаляя общности, считать $\|x\|=p x$. Для простоты, также не умаляя общности, положим $\alpha=1$. Зададимся некоторым малым $\varepsilon>0$. В $\left[{ }^{2}\right]$ доказано, что найдется натуральное $T(\varepsilon)$ такое, что если плановый горизонт $T \geqslant T(\varepsilon)$, то $\varrho(x(\tau), \hat{x})<\varepsilon$ для всех $\tau$ начиная с некоторого номера $\tau(\varepsilon)$.

Легко доказать, что для любого $\delta>0$ найдется натуральное число $N(\delta)$ такое, что $\varrho\left(A^{N(\delta)} x, \hat{x}\right)<\delta$ для любого $x \geqslant 0$. Известно, что норма матрицы $A^{t}$ стремится к единице при $t \rightarrow \infty$, поэтому существует $L$ такое, что $\left\|A^{t}\right\| \leqslant 2$ для * $t \geqslant L$. Положим $N=\max \{N(\varepsilon), L\}$. Будем далее считать, что $T \geqslant T^{\prime}=\max \{T(\varepsilon), N+1\}$.

Л е м а 1. Найдется константа $a>0$, не зависящая от выбора в такая, что для любого $x \geqslant 0$ выполняется $\varrho\left(A^{N} x, \hat{x}\right) \leqslant \operatorname{a\varepsilon \varrho ~}(x, \hat{x})$.

Д ок а з ательст во. Очевидно, что найдется положительная константа $b$ такая, что для любого вектора $y$ справедливо $\sum_{i=1}^{n}\left|y^{(i)}\right| \leqslant b\|y\|$. Обозначнм $m(\hat{x})=\min _{i=1, \ldots, n} \hat{x}^{(i)}, d=\varrho(x, \hat{x}), \beta(d)=1-b d / m(\hat{x}) . \quad$ Поскольку $\mathrm{Q}\left(A^{N} x, \hat{x}\right) \leqslant \varepsilon$ для всех $x \geqslant 0$, то, не умаляя общности, можно считать, что $d<m(\hat{x}) / 2 b$. В противном случае достаточно взять $a \geqslant 2 b / m(\hat{x})$.

Очевидно, вектор $x \geqslant 0$ можно представить в виде $x=$ $=\beta(d)\|x\| \hat{x}+e$, где $e \geqslant 0,\|e\|=b d\|x\| / m(\hat{x})$, так что $A^{N} x=\beta(d)\|x\| \hat{x}+$ $+A^{N} e$. Аналогично, так как $\mathrm{\varrho}\left(A^{N} e, \hat{x}\right) \leqslant \varepsilon$ и $\left\|A^{N}\right\| \leqslant 2$, то $A^{N} e=$ $=\beta(\varepsilon)\left\|A^{N} e\right\| \hat{x}+e^{\prime}$, где $e^{\prime} \geqslant 0, \quad\left\|e^{\prime}\right\|=b \varepsilon\left\|A^{N} e\right\| / m(\hat{x}) \leqslant b \varepsilon\left\|A^{N}\right\|\|e\| / m(\hat{x}) \leqslant$ $\leqslant 2 \varepsilon b^{2} d\|x\| /(m(\hat{x}))^{2}$. Итак, поскольку по соглашению норма аддитивна на $R_{+}{ }^{n}$ и $\beta(d)>1 / 2$, имеем

$$
\varrho\left(A^{N} x, \hat{x}\right)=\left\|\frac{\beta(d)\|x\| \hat{x}+\beta(\varepsilon)\left\|A^{N} e\right\| \hat{x}+e^{\prime}}{\beta(d)\|x\|+\beta(\varepsilon)\left\|A^{N} e\right\|+\left\|e^{\prime}\right\|}-\hat{x}\right\| \leqslant \frac{2\left\|e^{\prime}\right\|}{\beta(d)\|x\|} \leqslant \frac{8 \varepsilon b^{2} d}{(m(\hat{x}))^{2}},
$$

что и требовалось доказать.

Заметим, что упомянутую выше величину $N(\delta)$ можно определить жак функцию $N(\delta)=\min \left\{t \geqslant 0 \mid \sup _{x \geqslant 0} \varrho\left(A^{t} x, \hat{x}\right) \leqslant \delta\right\}$.

* Символом $\|\cdot\|$ будем пользоваться и для обозначения матричной нормы. 
Л ем м а $2 . \delta N(\delta) \underset{\delta \rightarrow 0}{\rightarrow \rightarrow 0}$.

Доказ ательство. Фиксируем некоторое значение $\delta_{0} \in(0, \varepsilon)$, причем $\delta_{0}<1 / a$, и положим $N_{0}=N\left(\delta_{0}\right)$. Для любого $x \geqslant 0$ имеем $\varrho\left(A^{N_{0}} x, \hat{x}\right) \leqslant \delta_{0}$, следовательно, по лемме $1 \varrho\left(A^{2 N_{0}} x, \hat{x}\right) \leqslant a \delta_{0}^{2}$ для $x \geqslant 0$, аналогично

$$
\mathrm{Q}\left(A^{k N_{0}} x, \hat{x}\right) \leqslant a^{k} \delta_{0}^{k+1} \text { для всех } x \geqslant 0, k=1,2, \ldots .
$$

Ясно, что для любого $\delta \in\left(0, \delta_{0}\right)$ найдется натуральное $k(\delta)$ такое, что $\delta \in\left(a^{k(\delta)} \delta_{0}^{k(\delta)+1}, a^{k(\delta)-1} \delta_{0}^{k(\delta)}\right]$.

Тем самым, по определению $N(\delta)$ и в силу (4), $\quad N(\delta) \leqslant k(\delta) N_{0}$. Таким образом, $\delta N(\delta) \leqslant$ $\leqslant a^{k(\delta)-1} \delta_{0}^{k(\delta)} k(\delta) N_{0}$. Стремление $\delta$ к нулю означает, что соответствующее $k(\delta) \rightarrow \infty$. Отсюда, поскольку $\delta_{0}<1 / a$, имеем $\delta N(\delta) \underset{8 \rightarrow 0}{\rightarrow} 0$, что и требовалось доказать.

Доказательство теоремы. Обозначим $\sigma=a b \varepsilon / \min \hat{x^{(i)}}$. Не умаляя общности, предположим, что исходное $\varepsilon$ выбрано настолько малым, что (по лемме 2)

$$
\sigma N \leqslant 1 / 32 \text {. }
$$

Вернемся к рассмотрению траектории $\{x(\tau)\}$. Возьмем произвольный момент $t \geqslant \tau(\varepsilon)$ и положим $d_{0}=\max _{s=t, \ldots, t+N-1} \mathrm{~g}(x(s), \hat{x})$. Как отмечалось, $d_{0} \leqslant \varepsilon$. Пусть $\gamma(t)$ - максимальное значение функционала в задаче (2) с начальным запасом, равным $x(t)$, так что соответствующий оптимальный выпуск в конце планового периода $x_{T}(t) \geqslant \gamma(t) x(t)$. Отсюда, по определению скользящего плана, в силу (1), леммы 1 и так как $\|x\|=p x$, имеем

$$
x(t+1) \geqslant A^{T-1} x_{T}(t) \geqslant \gamma(t) A^{T-1} x(t) \geqslant\left(1-\sigma d_{0}\right) \gamma(t)(p x(t)) \hat{x} .
$$

Рассмотрим точку $y_{T}=q(x(t)) x(t) / p x(t)$. Ясно, что в нее можно за $T$ шагов попасть из точки $y=A^{T} y_{T}$. Поскольку $\varrho\left(y_{T}, \hat{x}\right)=\varrho(x(t), \hat{x}) \leqslant d_{0}$, то по лемме $1 \mathrm{\varrho}(y, \hat{x}) \leqslant a \varepsilon d_{0}$. Отсюда, как легко показать, $y \leqslant$ $\leqslant\left(1+\sigma d_{0}\right)(p y) \hat{x}$. Так как $p-$ собственный вектор матрицы $A$, то $p y=p y_{T}=q(x(t)) \quad$ и, значит, $y \leqslant\left(1+\sigma d_{0}\right) q(x(t)) \hat{x} . \quad$ Поскольку $x(t) \geqslant \dot{q}(x(t)) \hat{x}$, то отсюда и из вида модели (1) следует, что из $x(t)$ можно за $T$ шагов попасть в точку $y^{T} /\left(1-\sigma d_{0}\right)=q(x(t)) x(t) /$ $\operatorname{lpx}(t)\left(1+\sigma d_{0}\right)$. Следовательно, по оптимальности имеем

$$
\gamma(t) \geqslant q(x(t)) / p x(t)\left(1+\sigma d_{0}\right) \geqslant q(x(t))\left(1-\sigma d_{0}\right) / p x(t) .
$$

Таким образом, в силу (6) и поскольку $q(\hat{x})=1$, верно $q(x(t+1)) \geqslant$ $\geqslant\left(1-\sigma d_{0}\right)^{2} q(x(t))$. Повторяя рассуждения применительно к моментам $t+1, \ldots, t+N-1$, получим

$$
q(x(t+N)) \geqslant\left(1-\sigma d_{0}\right)^{2 N} q(x(t)) \geqslant\left(1-2 \sigma N d_{0}\right) q(x(t))
$$

для всех $t \geqslant \tau(\varepsilon)$. Последнее в цепочке неравенств (7) следует из (5) и сравнения производных по $\Delta$ функций $(1-\Delta)^{N}$ и $1-N \Delta$, равных между собой при $\Delta=0$. 
Положим $z=A^{N} x(t+N), d_{1}=\varrho(x(t+N), \hat{x})$, так что по лемме 1 $\varrho(z, \hat{x}) \leqslant a d_{1} \varepsilon$, тем самым $z \geqslant\left(1-\sigma d_{1}\right)(p z) \hat{x}$ и $q(z) \geqslant\left(1-\sigma d_{1}\right) p z$. Следовательно, учитывая $(5), p z \leqslant q(z) /\left(1-\sigma d_{1}\right) \leqslant q(z)\left(1+2 \sigma d_{1}\right)$. В силу (1) $z \leqslant x(t)$, так что $q(z) \leqslant q(x(t))$. Таким образом, справедливо

$$
p z \leqslant q(x(t))\left(1+2 \sigma d_{1}\right) .
$$

Заметим, что $q(x) \leqslant p x$, поскольку $q(x) \hat{x} \leqslant x$ и $p \hat{x}=1$. Отсюда, так как мы положили $\|x\|=p x$ для $x \geqslant 0$, и по неравенству треугольника имеем

$$
\begin{gathered}
d_{1}=\frac{\|x(t+N)-p x(t+N) \hat{x}\|}{p x(t+N)} \leqslant \\
\leqslant \frac{\|x(t+N)-q(x(t+N)) \hat{x}\|+p x(t+N)-q(x(t+N))}{p x(t+N)}=! \\
=\frac{2[p x(t+N)-q(x(t+N))]}{p x(t+N)}=\frac{2[p z-q(x(t+N))]}{p x(t+N)},
\end{gathered}
$$

Отсюда, в силу (8), (7) и (5) следует

$$
\begin{aligned}
d_{1} & \leqslant \frac{2\left[q(x(t))\left(1+2 \sigma d_{1}\right)-q(x(t))\left(1-2 \sigma N d_{0}\right)\right]}{q(x(t))\left(1-2 \sigma N d_{0}\right)} \leqslant \\
& \leqslant 8\left(\sigma d_{1}+\sigma N d_{0}\right)<d_{1} / 2+d_{0} / 4
\end{aligned}
$$

так что $d_{1} \leqslant d_{0} / 2$, т. е.

$$
\mathrm{Q}(x(t+N), \hat{x}) \leqslant \max _{s=t, \ldots, t+N-1} \varrho(x(s), \hat{x}) / 2 .
$$

Поскольку это неравенство справедливо для всех $t \geqslant \tau(\varepsilon)$, то последовательно для $r=0,1, \ldots, N-1$ легко показать, что $\mathrm{\varrho}(x(t+r+N), \hat{x}) \leqslant d_{0} / 2 . \quad$ Таким образом, $\varrho(x(t+2 N), \hat{x}) \leqslant$ $\leqslant \max _{s=t+N, \ldots, t+2 N-1} \varrho(x(s), \hat{x}) / 2 \leqslant d_{0} / 4$. Аналогично, $\varrho(x(t+k N+r), \hat{x}) \leqslant$ $\leqslant d_{0} / 2^{k}$ для $t \geqslant \tau(\varepsilon), k=0,1, \ldots$, или

$$
\varrho(x(\tau), \hat{x}) \leqslant d_{0} / 2^{[(\tau-t) / N]} \leqslant \varepsilon / 2^{[(\tau-t) / N]}, \quad \tau \geqslant t \geqslant \tau(\varepsilon),
$$

где [·] - обозначение целой части числа. Следовательно, применяя формулу (7), которая в силу произвольности выбора $t \geqslant t(\varepsilon)$ означает, что для любого $\tau \geqslant \tau(\varepsilon)+N$ выполняется

$$
q(x(\tau)) \geqslant\left(1-2 \sigma N \max _{s=\tau-N, \ldots, \tau-1} \varrho(x(s), \hat{x})\right) q(x(\tau-N)),
$$

получим

$$
q(x(\tau)) \geqslant \prod_{k=0}^{[(\tau-\tau(\varepsilon)) / N]-1}\left(1-2 \sigma N \varepsilon / 2^{k}\right) q(x(t(\varepsilon))), \quad \tau \geqslant \tau(\varepsilon) .
$$


Легко видеть, что при $\tau \rightarrow \infty$ произведение сходится к положительной величине. Тем самым рассматриваемая траектория скользящего планирования строго отделена от нуля, что вместе с (9) доказывает теорему. 3 а м е ч н ие. Утверждение теоремы сохраняет силу, если в задаче (2) ограничение на пропорции выпуска в конечный момент имеет вид $x_{T}(k) \geqslant \gamma x_{s}(k-1)$ для некоторого $s=l, \ldots, T-1$, т. е. на каждой итерации пропорции фиксируются на уровне, соответствующем плану предыдущей итерации на $s$-й год, где $l$ - натуральное число, завнсящее только от матрицы $A$. Доказательство этого факта основывается на тех же соображениях, что и доказательство теоремы.

Полученные результаты можно интерпретировать следующим образом: сходимость процесса скользящего планирования можно обеспечить, если на каждой его итерации учитывать опыт, полученный на предыдущей итерации.

\section{Л ИТ Р АТ У РА}

1. Березнев а Т. Д., Экономика и матем. методы, 12, № 4, 740-746 (1976).

2. К а г а н о в и ч М., Изв. АН ЭССР, Физ. Матем., 28, № 4, 310-316 (1979).

Ннститут кибернетики . Академии наук Эстонской ССР
Поступила в редакцию $24 / \mathrm{X} 1979$

\section{KAGANOVITS}

\section{LIBISEVA PLANEERIMISE TRAJEKTOORI KOONDUVUSEST MAJANDUSHARUDEVAHELISES DUNAAMILISES MUDELIS}

Artiklis on uuritud libiseva planeerimise trajektooride asümptootikat ühes majandusliku kasvu maatriksmudelis. On teada, et need trajektoorid üldiselt ei koondu statsionaarseks seisuks. Töös on koonduvus tõestatud, kusjuures on kasutatud mõningaid eespool mainitud trajektooride konstrueerimise printsiibi kohta käivaid lisatingimusi.

\section{KAGANOVICH}

\section{ON A CONVERGENCE OF THE SLIDING PLANNING PATHS IN A MULTISECTOR DYNAMIC MODEL}

The asymptotic behavior of sliding planning paths in the von Neumann model with no joint production is considered. According to the principle of sliding planning, the plan is revised at each year of planning interval, an additional year beimg appended to the last. More exactly, let $x(k)$ be the sliding plan for the year $k$, then the plan $x(k+1)$ for the next year is defined as follows. An optimal path $\left\{x_{t}(k)\right\}_{t=0}^{T}$ is computed, taking the current state for the initial point: $x_{0}(k)=x(k)$, the planning horizon $T$ remaining constant for all the periods, and the (final-state) objective functions being allowed to vary. Then the first year optimal plan $x_{t}(k)$ is taken for $x(k+1)$.

Under the strong turnpike theorem assumptions it was shown in $\left.{ }^{2}\right]$ for the more general von Neumann-Gale model that, beginning with some moment, the sliding planning paths lie close to the von Neumann ray. However, an example demonstrates that they need not in general converge to it.

Our intent is to determine such conditions which would ensure the convergence. Here the convergence of the sliding planning paths is proved under a restriction imposed on the type of optimal paths from which they are being constructed (see formula (2)). This restriction may be interpreted as follows: every iteration of the sliding planning proceeds in accordance with the experience derived from the previous one. 